\title{
CONVOLUTION OF ORBITAL MEASURES IN SYMMETRIC SPACES
}

\author{
BOUDJEMÂA ANCHOUCHE and SANJIV KUMAR GUPTA $\bowtie$
}

(Received 5 September 2010)

\begin{abstract}
Let $G / K$ be a noncompact symmetric space, $G_{c} / K$ its compact dual, $\mathfrak{g}=\mathfrak{k} \oplus \mathfrak{p}$ the Cartan decomposition of the Lie algebra $\mathfrak{g}$ of $G, \mathfrak{a}$ a maximal abelian subspace of $\mathfrak{p}, H$ be an element of $\mathfrak{a}, a=\exp (H)$, and $a_{c}=\exp (i H)$. In this paper, we prove that if for some positive integer $r, v_{a_{c}}^{r}$ is absolutely continuous with respect to the Haar measure on $G_{c}$, then $v_{a}^{r}$ is absolutely continuous with respect to the left Haar measure on $G$, where $v_{a_{c}}$ (respectively $v_{a}$ ) is the $K$-bi-invariant orbital measure supported on the double coset $K a_{c} K$ (respectively $K a K$ ). We also generalize a result of Gupta and Hare ['Singular dichotomy for orbital measures on complex groups', Boll. Unione Mat. Ital. (9) III (2010), 409-419] to general noncompact symmetric spaces and transfer many of their results from compact symmetric spaces to their dual noncompact symmetric spaces.
\end{abstract}

2010 Mathematics subject classification: primary 43A80; secondary 58C35, 53C35.

Keywords and phrases: convolution, double coset, orbital measures, symmetric spaces.

\section{Introduction}

In [1], Dunkl proved that the convolution of the surface measure of the sphere in $\mathbb{R}^{n}$ with itself is absolutely continuous with respect to the Lebesgue measure in $\mathbb{R}^{n}$. Ragozin [11, 12] generalized Dunkl's result to the setup of symmetric spaces; more precisely, he proved [12, Theorem 2.5] that if $G / K$ is a Riemannian symmetric space and $\left\{v_{i}\right\}_{i=1}^{\operatorname{dim} G / K}$ are $K$-bi-invariant continuous zonal measures on $G$, then the convolution $v_{1} * \cdots * v_{\operatorname{dim} G / K}$ is absolutely continuous with respect to the left Haar measure on $G$, and he conjectured [12, p. 375] that $\operatorname{dim} G / K$ may be improved to $[(\operatorname{dim} G / K-1) / j]+1$, where $j$ is the minimum dimension of the nonfinite $K$-orbits in $G / K$. In a series of papers, Hare and the second author of this paper have partially improved Ragozin's result mentioned above. In [6, Theorem 3.1] they proved that if $a \in \mathrm{SU}(n) \backslash N_{\mathrm{SU}(n)}(\mathrm{SO}(n))$, where $N_{\mathrm{SU}(n)}(\mathrm{SO}(n))$ is the normalizer of $\mathrm{SO}(n)$ in $\mathrm{SU}(n)$, and $v_{a}=m_{\mathrm{SO}(n)} * \delta_{a} * m_{\mathrm{SO}(n)}$, where $m_{\mathrm{SO}(n)}$ denotes the Haar measure on $\mathrm{SO}(n)$ and $\delta_{a}$ denotes the point mass at $a$, then $v_{a}^{n}$ is absolutely continuous

The authors are grateful to Sultan Qaboos University for its support.

(c) 2011 Australian Mathematical Publishing Association Inc. 0004-9727/2011 \$16.00 
with respect to the Haar measure on $\mathrm{SU}(n)$. Moreover, they proved that there exists $a \in \mathrm{SU}(n) \backslash N_{\mathrm{SU}(n)}(\mathrm{SO}(n))$ such that $v_{a}^{n-1}$ is singular with respect to the Haar measure on $\mathrm{SU}(n)$. Note that $\operatorname{dim} \mathrm{SU}(n) / \mathrm{SO}(n)=\left(n^{2}+n-2\right) / 2$. In [4, Theorem 3.1] they proved that for a compact symmetric space $G_{c} / K$, there is a dense subset $D \subseteq G_{c}$ such that if $a_{1}, a_{2} \in D$, then $v_{a_{1}} * v_{a_{2}}$ is absolutely continuous with respect to Haar measure on $G_{c}$. Finally, in [2, Theorem 4.1], they proved if $G_{c}$ is a compact, connected, simple, classical Lie group, $G_{c}^{\mathbb{C}}$ the complexification of $G_{c}, \mathfrak{g}_{c}$ the Lie algebra of $G_{c}$, and $H \in \mathfrak{g}_{c}$ is such that $a=\exp (i H) \in G_{c}^{\mathbb{C}} \backslash N_{G_{c}^{\mathbb{C}}}\left(G_{c}\right)$, then $v_{a}$ is absolutely continuous with respect to the left Haar measure on $G_{c}^{\mathbb{C}}$ if and only if $\mu_{H}^{r}$ is absolutely continuous with respect to the Lebesgue measure on $\mathfrak{g}_{c}$, where $\mu_{H}$ is the $G_{c}$-invariant orbital measure supported on the adjoint orbit of $H$ in $\mathfrak{g}_{c}$. The aim of this paper is to extend some of the results mentioned above.

Our main theorem, Theorem 3.1 below, is an extension of Gupta and Hare's result in [2, Theorem 4.1] to general symmetric spaces (the notation is as in Section 2).

Combining the main theorem with some results of Hare and the second author, which were mentioned above, we obtain several interesting results, which will be listed in Section 4. In order not to duplicate the results, we will skip those results and invite the interested reader to go directly to that section.

This paper is organized as follows. In Section 2 we collect some basic facts about symmetric spaces, restricted roots, and orbital measures. In Section 3 we prove the main theorem. In Section 4 we extend some of the results obtained in $[4,6]$ to the noncompact symmetric spaces using the main theorem. In the final section we show that the inequality of the general transference result Theorem 3.1(1) can be strict and suggest some open problems.

\section{Notation and basic facts}

Let $M$ be a symmetric space of noncompact type and let $G$ be the connected component of the isometry group of $M$ which contains the identity. Then $G$ is a semisimple Lie group with trivial center which acts transitively on $M$. Fix a point $p \in M$ and let $K$ be the subgroup of $G$ that fixes $p$. Then $K$ is a compact subgroup of $G$ and $M$ is diffeomorphic to $G / K$ via the map

$$
\begin{aligned}
\Psi: G / K & \longrightarrow M \\
g K & \longmapsto g \cdot p .
\end{aligned}
$$

Throughout what follows we will identify $M$ with $G / K$, and put $M=G / K$. Let $\mathfrak{g}$ be the Lie algebra of $G, \theta: \mathfrak{g} \longrightarrow \mathfrak{g}$ be a Cartan involution, and let

$$
\mathfrak{k}=\{X \in \mathfrak{g} \mid \theta(X)=X\} \quad \text { and } \quad \mathfrak{p}=\{X \in \mathfrak{g} \mid \theta(X)=-X\} .
$$

The decomposition

$$
\mathfrak{g}=\mathfrak{k} \oplus \mathfrak{p}
$$


is called the Cartan decomposition of the Lie algebra $\mathfrak{g}$. It is easy to check that

$$
[\mathfrak{k}, \mathfrak{k}] \subseteq \mathfrak{k}, \quad[\mathfrak{k}, \mathfrak{p}] \subseteq \mathfrak{p}, \quad[\mathfrak{p}, \mathfrak{p}] \subseteq \mathfrak{k} .
$$

Inside the complexification $\mathfrak{g}^{\mathbb{C}}$ of $\mathfrak{g}$ we consider the subset

$$
\mathfrak{g}_{c}=\mathfrak{k} \oplus i \mathfrak{p} .
$$

The Lie bracket of $\mathfrak{g}$ induces a Lie bracket on the complexification on $\mathfrak{g}^{\mathbb{C}}$, and therefore $\mathfrak{g}_{c}$ inherits a Lie bracket from $\mathfrak{g}^{\mathbb{C}}$, which makes $\mathfrak{g}_{c}$ a real subalgebra of $\mathfrak{g}^{\mathbb{C}}$. Let $G_{c}$ be a Lie group with Lie algebra $\mathfrak{g}_{c}$. Then $G_{c}$ is a compact Lie group and $M_{c}=G_{c} / K$ is a compact symmetric space, called the compact dual of $M=G / K$.

Fix a maximal abelian (as a subalgebra of $\mathfrak{g}$ ) subspace $\mathfrak{a}$ of $\mathfrak{p}$, and denote by $\mathfrak{a}^{*}$ its dual. For $\alpha \in \mathfrak{a}^{*}, \alpha \neq 0$, consider the set

$$
\mathfrak{g}_{\alpha}=\{X \in \mathfrak{g} \mid[H, X]=\alpha(H) X \text { for all } H \in \mathfrak{a}\} .
$$

The set $\Sigma$ of restricted roots is defined by

$$
\Sigma=\left\{\alpha \in \mathfrak{a}^{*} \mid \mathfrak{g}_{\alpha} \neq 0\right\} .
$$

Then it is very well known that $\Sigma$ is finite and nonempty, and we have the decomposition

$$
\mathfrak{g}=\mathfrak{g}_{0} \oplus \sum_{\alpha \in \Sigma} \mathfrak{g}_{\alpha}
$$

where

$$
\mathfrak{g}_{0}=\{X \in \mathfrak{g} \mid[H, X]=0 \text { for all } H \in \mathfrak{a}\}=\mathfrak{a} \oplus \mathfrak{m},
$$

with

$$
\mathfrak{m}=Z_{\mathfrak{k}}(\mathfrak{a})=\{X \in \mathfrak{k} \mid[X, Y]=0, \text { for all } Y \in \mathfrak{a}\} .
$$

It is easy to check that

$$
\left[\mathfrak{g}_{\alpha}, \mathfrak{g}_{\beta}\right] \subseteq \mathfrak{g}_{\alpha+\beta}, \quad \theta \mathfrak{g}_{\alpha}=\mathfrak{g}_{-\alpha}, \quad\left[X_{\alpha}, \theta X_{\alpha}\right]=B\left(X_{\alpha}, \theta X_{\alpha}\right) H_{\alpha},
$$

where $H_{\alpha}$ is defined by $B\left(H, H_{\alpha}\right)=\alpha(H)$, in which $B$ is the Killing form.

Clearly, we have

$$
\mathfrak{k}=\operatorname{span}\left\{X+\theta X \mid X \in \mathfrak{g}_{\alpha}, \alpha \in \Sigma\right\} \oplus \mathfrak{m} .
$$

and

$$
\mathfrak{p}=\operatorname{span}\left\{X-\theta X \mid X \in \mathfrak{g}_{\alpha}, \alpha \in \Sigma\right\} \oplus \mathfrak{a} .
$$

Since on a compact Lie group left Haar measures coincide with right Haar measures, and since all left Haar measures are, up to a constant, the same on a fixed Lie group, throughout the paper we will fix Haar measures on $G_{c}$ and $K$ and a left Haar measure on $G$ and we will talk of 'the' Haar measure on $G_{c}$ and $K$ and 'the' left Haar measure on $G$. A basic reference on symmetric spaces is [8]. 
2.1. Compact Lie groups seen as symmetric spaces. Let $G_{c}$ be a compact Lie group and consider the map

$$
\begin{aligned}
\theta: G_{c} \times G_{c} & \longrightarrow G_{c} \times G_{c} \\
\left(g_{1}, g_{2}\right) & \longmapsto\left(g_{2}, g_{1}\right) .
\end{aligned}
$$

Then $\theta$ is an involution, that is, $\theta^{2}=\mathrm{id}$, with fixed point set

$$
\Delta_{G_{c}}=\left\{(g, g) \mid g \in G_{c}\right\},
$$

which can be clearly identified with $G_{c}$. Then the compact group $G_{c}$ can be identified with the symmetric space $\left(G_{c} \times G_{c}\right) / \Delta_{G_{c}}$ via the map

$$
\begin{aligned}
\rho: G_{c} & \longrightarrow\left(G_{c} \times G_{c}\right) / \Delta_{G_{c}} \\
g & \longmapsto(g, e) \Delta_{G_{c}},
\end{aligned}
$$

which is easily seen to be a diffeomorphism.

Now let us describe the dual of $G_{c}$, seen as a symmetric space. Put $\widetilde{G}=G_{c} \times G_{c}$ and let $\mathfrak{g}_{c}$ (respectively $\widetilde{\mathfrak{g}}$ ) be the Lie algebra of $G_{c}$ (respectively $\widetilde{G}$ ). Then the decomposition of $\tilde{\mathfrak{g}}$ under the action of $d \theta$ is given by

$$
\tilde{\mathfrak{g}}=\mathfrak{k} \oplus \mathfrak{p}
$$

where

$$
\mathfrak{k}=\Delta \mathfrak{g}_{c}=\left\{(X, X) \mid X \in \mathfrak{g}_{c}\right\} \quad \text { and } \quad \mathfrak{p}=\left\{(X,-X) \mid X \in \mathfrak{g}_{c}\right\} .
$$

Let

$$
\tilde{\mathfrak{g}}_{\text {dual }}=\mathfrak{k} \oplus i \mathfrak{p} .
$$

Then $\widetilde{\mathfrak{g}}_{\text {dual }}$ is a real Lie subalgebra of the complexification

$$
\widetilde{\mathfrak{g}}^{\mathbb{C}}=\tilde{\mathfrak{g}} \oplus i \widetilde{\mathfrak{g}}
$$

of the Lie algebra $\tilde{\mathfrak{g}}$. It is easy to prove that $\tilde{\mathfrak{g}}_{\text {dual }}$ is isomorphic to $\mathfrak{g}_{c}^{\mathbb{C}}$ via the map

$$
\begin{gathered}
\sigma: \tilde{\mathfrak{g}}_{\text {dual }}=\mathfrak{k} \oplus i \mathfrak{p} \longrightarrow \mathfrak{g}_{c}^{\mathbb{C}} \\
(X, X)+i(Y,-Y) \longmapsto X+i Y .
\end{gathered}
$$

Then the Lie group with Lie algebra $\widetilde{\mathfrak{g}}_{\text {dual }}$ can be identified with the complexification $G_{c}^{\mathbb{C}}$ of $G_{c}$. Consider the involution

$$
\begin{aligned}
\tau: \mathfrak{g}_{c}^{\mathbb{C}}=\mathfrak{g}_{c} \oplus i \mathfrak{g}_{c} & \longrightarrow \mathfrak{g}_{c}^{\mathbb{C}} \\
X+i Y & \longmapsto X-i Y,
\end{aligned}
$$

and denote also by $\tau$ the involution on $G_{c}^{\mathbb{C}}$ induced by the involution $\tau$ of $\mathfrak{g}_{c}^{\mathbb{C}}$. The fixed point of $\tau$ on $G_{c}^{\mathbb{C}}$ is $G_{c}$. Therefore $G_{c}^{\mathbb{C}} / G_{c}$ is a symmetric space, dual of the compact Lie group $G_{c}$, seen as a symmetric space. For example, the dual of $\mathrm{SU}(n)$, seen as a symmetric space, is $\operatorname{SL}(n, \mathbb{C}) / \mathrm{SU}(n)$. Let

$$
C_{a}=\left\{\mathrm{gag}^{-1} \mid g \in G_{c}\right\}
$$

be the orbit of an element $a \in G_{c}$ under conjugation. Under the diffeomorphism (2.1), $C_{a}$ corresponds to the double coset $\Delta_{G}(a, e) \Delta_{G}$, which we will identify (after identifying $\Delta_{G_{c}}$ with $G_{c}$ ), with $G_{c}(a, e) G_{c}$. 
2.2. Orbital measures. Before introducing the orbital measures which will be of interest in this paper, let us first recall the definition of the convolution of positive measures on a Lie group. Let $v_{1}, \ldots, v_{r}$ be $r$ positive measures on a Lie group $G$ (actually $G$ need only be a locally compact group). Then the convolution $v_{1} * \cdots * v_{r}$ of the measures $v_{1}, \ldots, v_{r}$ is defined by

$$
\begin{aligned}
\left\langle v_{1} * \cdots * v_{r}, f\right\rangle & =\int_{G} f(g) d\left(v_{1} * \cdots * v_{r}\right)(g) \\
& =\int_{G} \cdots \int_{G} f\left(g_{1} \cdots g_{r}\right) d v_{1}\left(g_{1}\right) \cdots d v_{r}\left(g_{r}\right) ; \quad f \in C_{c}(G) .
\end{aligned}
$$

If $v_{i}$ is supported on $S_{i}, i=1, \ldots r$, then the measure $v_{1} * \cdots * v_{r}$ is supported on $S_{1} \cdots S_{r}$, and in this case

$$
\begin{aligned}
\left\langle v_{1} * \cdots * v_{r}, f\right\rangle=\int_{S_{1}} d v_{1}\left(g_{1}\right) \int_{S_{2}} d \nu_{2}\left(g_{2}\right) & \\
& \cdots \int_{S_{r}} f\left(g_{1} \cdots g_{r}\right) d v_{r}\left(g_{r}\right) ; \quad f \in C_{c}(G) .
\end{aligned}
$$

We now define four different types of orbits and continuous singular measures supported on them.

(1) Fix a maximal torus $T$ in $G_{c}$. Let $h \in G_{c}$, and let $C_{h}$ be the conjugacy class containing $h$, that is,

$$
C_{h}=\left\{g h g^{-1}: g \in G_{c}\right\} \text {. }
$$

Every conjugacy class contains an element in the maximal torus $T$. Conjugacy classes are homogeneous submanifolds of proper dimension in $G_{c}$ (as their codimension in $G_{c}$ is at least $\operatorname{rank}\left(G_{c}\right)$ ) and thus have Haar measure zero.

For an element $h \in G_{c}$, the orbital measure, $v_{h}$, supported on $C_{h}$, is the $G_{c^{-}}$ invariant measure defined by

$$
\begin{aligned}
\left\langle v_{h}, f\right\rangle & =\int_{G_{c}} f d v_{h} \\
& =\int_{G_{c}} f\left(g h g^{-1}\right) d m_{G_{c}}(g) ; \quad f \in C\left(G_{c}\right)
\end{aligned}
$$

where $m_{G_{c}}$ is the Haar measure on $G_{c}$. Orbital measures $v_{h}$ are singular (with respect to the Haar measure $m_{G_{c}}$ on $G_{c}$ [10]), probability measures, and are continuous measures if and only if $h$ does not belong to the center $Z\left(G_{c}\right)$ of $G_{c}$. They are also central measures, meaning they commute with all other measures under convolution.

(2) The group $G_{c}$ acts on its Lie algebra $\mathfrak{g}_{c}$ by the adjoint action. Let $H \in \mathfrak{g}_{c}$ and $O_{H}$ denote its adjoint orbit,

$$
O_{H}=\left\{\operatorname{Ad}(g) H: g \in G_{c}\right\}
$$


Let $\mathfrak{t}$ be the Lie algebra of the maximal torus $T$ mentioned above. Every adjoint orbit $O_{H}$ contains an element in the Cartan subalgebra $\mathfrak{t}$ of the Lie algebra $\mathfrak{g}_{c}$. Adjoint orbits are submanifolds of proper dimension in $\mathfrak{g}_{c}$ [13] (as their codimension in $\mathfrak{g}_{c}$ is at least $\operatorname{rank}\left(\mathfrak{g}_{c}\right)$ and so have the Lebesgue measure zero.

For any $H \in \mathfrak{g}_{c}$, the $G_{c}$ invariant orbital measure $\mu_{H}$, supported on $O_{H}$, is given by

$$
\begin{aligned}
\left\langle\mu_{H}, f\right\rangle & =\int_{\mathfrak{g}_{c}} f d \mu_{H} \\
& =\int_{G_{c}} f(\operatorname{Ad}(g) H) d m_{G_{c}}(g) ; \quad f \in C\left(\mathfrak{g}_{c}\right) .
\end{aligned}
$$

Orbital measures $\mu_{H}$ are singular (with respect to Lebesgue measure on $\mathfrak{g}_{c}$ ) and are continuous measures if and only if $H \neq 0$.

(3) Let $H \in \mathfrak{p}$. The orbit of $H$ under the adjoint action of $K$ on $\mathfrak{p}$, denoted by $O_{K, H}$, is given by

$$
O_{K, H}=\{\operatorname{Ad}(k) H \mid k \in K\} .
$$

Since $\mathfrak{p}=\bigcup_{k \in K} \operatorname{Ad}(k) \mathfrak{a}$, every orbit $O_{K, H}$ contains an element of a. Orbits $O_{K, H}$ are of proper dimension in $\mathfrak{p}$ (as their co-dimension in $\mathfrak{p}$ is greater than or equal to the rank of the noncompact symmetric space $M$ ).

For $H \in \mathfrak{p}$, define a $K$-invariant measure $\mu_{K, H}$ supported on $O_{K, H}$ as follows: for $f \in C(\mathfrak{p})$, we put

$$
\left\langle\mu_{K, H}, f\right\rangle:=\int_{K} f(\operatorname{Ad}(g) H) d m_{K}(g),
$$

where $m_{K}$ is the Haar measure of $K$. It is clear that $\mu_{K, H}$ is a singular measure (with respect to Lebesgue measure on $\mathfrak{p}$ ) and is continuous if and only if $H \neq 0$.

(4) Let $G / K$ be a symmetric space (not necessarily noncompact). Consider the natural action of $K \times K$ on $G$ defined by

$$
\begin{aligned}
\chi:(K \times K) \times G & \longrightarrow G \\
\left(\left(k_{1}, k_{2}\right), a\right) & \longmapsto k_{1} a k_{2} .
\end{aligned}
$$

The orbit of a point $a \in G$ is the double coset space $K a K$, which we can assume without loss of generality to be of the form $K \exp (H) K$, for some $H \in \mathfrak{a}$ [9, p. 485]. Double cosets $K a K$ are of proper dimension in $G$ (as their co-dimension in $G$ is greater than or equal to the rank of the symmetric space $G / K)$. Each orbit is equipped with a unique $K \times K$ invariant (bi- $K$-invariant) measure $v_{a}$, defined as follows: for $f \in C(G)$, we put

$$
\begin{aligned}
\left\langle v_{a}, f\right\rangle & =\int_{G} f(g) d v_{a}(g) \\
& =\int_{K} \int_{K} f\left(k_{1} a k_{2}\right) d m_{K}\left(k_{1}\right) d m_{K}\left(k_{2}\right) .
\end{aligned}
$$


The set of the measures $v_{a}$ is a Banach algebra under convolution and is denoted by $M(K \backslash G / K)$. It can be seen that $v_{a}=m_{K} * \delta_{a} * m_{K}$, where $\delta_{a}$ denotes the point mass at $a$. It is clear that $v_{a}$ is singular with respect to the left Haar measure on the group $G$. Clearly, $v_{a}$ is continuous if and if only $a$ is not in the normalizer of $K$ in $G$.

\section{Convolution of orbital measures in symmetric spaces}

Let $M=G / K$ be a noncompact Riemannian symmetric space and $G_{c} / K$ its compact dual. In this section we give a proof of our main theorem which consists of two parts: the first describes a transference result from a compact symmetric space to its dual, and the second generalizes Gupta and Hare's result in [2, Theorem 4.1] to general symmetric spaces.

Theorem 3.1. Let $H$ be an element of $\mathfrak{a}, H \neq 0, a=\exp (H), a_{c}=\exp (i H)$, and let

$$
\begin{gathered}
n_{a} \stackrel{\text { def }}{=} \min \left\{r \in \mathbb{N} \mid v_{a}^{r} \ll m_{G}\right\}, \\
n_{a_{c}}:=\operatorname{def} \\
n(K, a) \stackrel{\text { def }}{:=} \min \left\{r \in \mathbb{N} \mid v_{a_{c}}^{r} \ll m_{G_{c}}\right\}, \\
\left.n \in \mathbb{N} \mid \mu_{K, H}^{r} \ll \mu_{\mathfrak{p}}\right\},
\end{gathered}
$$

where the symbol '《' means 'is absolutely continuous with respect to', and $m_{G}$ (respectively $m_{G_{c}} ; \mu_{\mathfrak{p}}$ ) denotes the left Haar measure on $G$ (respectively Haar measure on $G_{c}$; Lebesgue measure on $\left.\mathfrak{p}\right)$. Then:
(1) $n_{a} \leq n_{a_{c}}$;
(2) $n_{a}=n(K, a)$.

REMARK 3.2. In Section 5 it will be shown that the inequality in part (1) of Theorem 3.1 can be strict.

Before proving the theorem, we need the following preliminary results.

Lemma 3.3. Let $H \in \mathfrak{a}$, and let $X_{\alpha} \in \mathfrak{g}_{\alpha}$. Then:

(1) $\operatorname{Ad}(\exp (i H))\left(X_{\alpha} \pm \theta X_{\alpha}\right)=\cos (\alpha(H))\left(X_{\alpha} \pm \theta X_{\alpha}\right)+i \sin (\alpha(H))\left(X_{\alpha} \mp \theta X_{\alpha}\right)$;

(2) $\operatorname{Ad}(\exp (H))\left(X_{\alpha} \pm \theta X_{\alpha}\right)=\sinh (\alpha(H))\left(X_{\alpha} \mp \theta X_{\alpha}\right)+\cosh (\alpha(H))\left(X_{\alpha} \pm \theta X_{\alpha}\right)$.

PROOF. (1) From the identities

$$
\begin{aligned}
\operatorname{Ad}(\exp (i H))\left(X_{\alpha}\right) & =\exp (\operatorname{ad}(i H)) X_{\alpha}=e^{i \alpha(H)} X_{\alpha}, \\
\operatorname{Ad}(\exp (i H))\left(\theta X_{\alpha}\right) & =\exp (\operatorname{ad}(i H)) \theta X_{\alpha}=e^{-i \alpha(H)}\left(\theta X_{\alpha}\right),
\end{aligned}
$$

we deduce that

$$
\begin{aligned}
& \operatorname{Ad}(\exp (i H))\left(X_{\alpha} \pm \theta X_{\alpha}\right) \\
& \quad=\exp (\operatorname{ad}(i H))\left(X_{\alpha} \pm \theta X_{\alpha}\right)=e^{i \alpha(H)} X_{\alpha} \pm e^{-i \alpha(H)} \theta X_{\alpha} \\
& \quad=\cos (\alpha(H))\left(X_{\alpha} \pm \theta X_{\alpha}\right)+i \sin (\alpha(H))\left(X_{\alpha} \mp \theta X_{\alpha}\right) .
\end{aligned}
$$


(2) Similarly, from the identities

$$
\begin{aligned}
\operatorname{Ad}(\exp (H))\left(X_{\alpha}\right) & =e^{\alpha(H)} X_{\alpha}, \\
\operatorname{Ad}(\exp (H))\left(\theta X_{\alpha}\right) & =e^{-\alpha(H)}\left(\theta X_{\alpha}\right),
\end{aligned}
$$

we deduce that

$$
\begin{aligned}
\operatorname{Ad}(\exp (H))\left(X_{\alpha} \pm \theta X_{\alpha}\right) & =\exp (\operatorname{ad}(H))\left(X_{\alpha} \pm \theta X_{\alpha}\right) \\
& =e^{\alpha(H)} X_{\alpha} \pm e^{-\alpha(H)} \theta X_{\alpha} \\
& =\sinh (\alpha(H))\left(X_{\alpha} \mp \theta X_{\alpha}\right)+\cosh (\alpha(H))\left(X_{\alpha} \pm \theta X_{\alpha}\right) .
\end{aligned}
$$

This concludes the proof.

Put

$$
\begin{aligned}
\mathcal{V}\left(a_{c}, k_{2}, \ldots, k_{n}\right) & =\mathfrak{k}+\operatorname{Ad}\left(a_{c}\right) \mathfrak{k}+\operatorname{Ad}\left(a_{c} k_{2} a_{c}\right) \mathfrak{k}+\cdots+\operatorname{Ad}\left(a_{c} k_{2} a_{c} \cdots k_{n} a_{c}\right) \mathfrak{k}, \\
\mathcal{V}\left(a, k_{2}, \ldots, k_{n}\right) & =\mathfrak{k}+\operatorname{Ad}(a) \mathfrak{k}+\operatorname{Ad}\left(a k_{2} a\right) \mathfrak{k}+\cdots+\operatorname{Ad}\left(a k_{2} a \cdots k_{n} a\right) \mathfrak{k},
\end{aligned}
$$

and

$$
\begin{aligned}
\mathcal{W}\left(a_{c}, k_{2}, \ldots, k_{n}\right)=\mathfrak{k} & +i\left(\mathcal{T}_{a_{c}}+\operatorname{Ad}\left(a_{c} k_{2}\right) \mathcal{T}_{a_{c}}\right. \\
& \left.+\operatorname{Ad}\left(a_{c} k_{2} a_{c} k_{3}\right) \mathcal{T}_{a_{c}}+\cdots+\operatorname{Ad}\left(a_{c} k_{2} \cdots a_{c} k_{n}\right) \mathcal{T}_{a_{c}}\right), \\
\mathcal{W}\left(a, k_{2}, \ldots, k_{n}\right)=\mathfrak{k}+ & \mathcal{T}_{a}+\operatorname{Ad}\left(a k_{2}\right) \mathcal{T}_{a} \\
& +\operatorname{Ad}\left(a k_{2} a k_{3}\right) \mathcal{T}_{a}+\cdots+\operatorname{Ad}\left(a k_{2} \cdots a k_{n}\right) \mathcal{T}_{a},
\end{aligned}
$$

where

$$
\begin{aligned}
\mathcal{T}_{a_{c}} & =\operatorname{span}\left\{\left(X_{\alpha}-\theta X_{\alpha}\right) \mid \alpha \text { restricted root such that } \alpha(H) \neq 0(\bmod \pi)\right\}, \\
\mathcal{T}_{a} & =\operatorname{span}\left\{\left(X_{\alpha}-\theta X_{\alpha}\right) \mid \alpha \text { restricted root such that } \alpha(H) \neq 0\right\} .
\end{aligned}
$$

PROPOSITION 3.4. Let $\mathcal{V}\left(a_{c}, k_{2}, \ldots, k_{n}\right)$ and $\mathcal{W}\left(a_{c}, k_{2}, \ldots, k_{n}\right)$ be as above. Then:

(1) $\mathcal{V}\left(a_{c}, k_{2}, \ldots, k_{n}\right)=\mathcal{W}\left(a_{c}, k_{2}, \ldots, k_{n}\right)$;

(2) $\mathcal{V}\left(a, k_{2}, \ldots, k_{n}\right)=\mathcal{W}\left(a, k_{2}, \ldots, k_{n}\right)$.

ProOF. Let $\alpha \in \Sigma$ and $M \in \mathfrak{m}$. From Lemma 3.3(1),

$$
\begin{aligned}
\operatorname{Ad}(\exp (i H))\left(X_{\alpha}+\theta X_{\alpha}+M\right)= & \cos (\alpha(H))\left(X_{\alpha}+\theta X_{\alpha}\right) \\
& +i \sin (\alpha(H))\left(X_{\alpha}-\theta X_{\alpha}\right) .
\end{aligned}
$$

Hence

$$
\operatorname{Ad}\left(a_{c}\right) \mathfrak{k} \subseteq \mathfrak{k}+i \mathcal{T}_{a_{c}}
$$

Consequently

$$
\begin{aligned}
\operatorname{Ad}\left(a_{c} k_{2} a_{c}\right) \mathfrak{k} & \subseteq \operatorname{Ad}\left(a_{c} k_{2}\right)\left(\mathfrak{k}+i \mathcal{T}_{a_{c}}\right) \\
& \subseteq \mathfrak{k}+i\left(\mathcal{T}_{a_{c}}+\operatorname{Ad}\left(a_{c} k_{2}\right)\left(\mathcal{T}_{a_{c}}\right)\right)
\end{aligned}
$$


By induction we get

$$
\mathcal{V}\left(a_{c}, k_{2}, \ldots, k_{n}\right) \subseteq \mathcal{W}\left(a_{c}, k_{2}, \ldots, k_{n}\right)
$$

Again, from Lemma 3.3,

$$
\begin{aligned}
i \sin (\alpha(H))\left(X_{\alpha}-\theta X_{\alpha}\right)=- & \cos (\alpha(H))\left(X_{\alpha}+\theta X_{\alpha}\right) \\
& +\operatorname{Ad}(\exp (i H))\left(X_{\alpha}+\theta X_{\alpha}\right) .
\end{aligned}
$$

Therefore

$$
\mathfrak{k}+i \mathcal{T}_{a_{c}} \subseteq \mathfrak{k}+\operatorname{Ad}\left(a_{c}\right) \mathfrak{k} .
$$

From (3.3), we deduce that

$$
\begin{aligned}
& i \sin (\alpha(H)) \operatorname{Ad}\left(a_{c} k_{2}\right)\left(X_{\alpha}-\theta X_{\alpha}\right) \\
& \quad=\operatorname{Ad}\left(a_{c} k_{2}\right)\left[-\cos (\alpha(H))\left(X_{\alpha}+\theta X_{\alpha}\right)+\operatorname{Ad}(\exp (i H))\left(X_{\alpha}+\theta X_{\alpha}\right)\right] .
\end{aligned}
$$

Hence

$$
i \operatorname{Ad}\left(a_{c} k_{2}\right) \mathcal{T}_{a_{c}} \subseteq \operatorname{Ad}\left(a_{c}\right) \mathfrak{k}+\operatorname{Ad}\left(a_{c} k_{2} a_{c}\right) \mathfrak{k} .
$$

Then

$$
\mathcal{W}\left(a_{c}, k_{2}\right) \subseteq \mathcal{V}\left(a_{c}, k_{2}\right)
$$

By induction,

$$
\mathcal{W}\left(a_{c}, k_{2}, \ldots, k_{n}\right) \subseteq \mathcal{V}\left(a_{c}, k_{2}, \ldots, k_{n}\right) .
$$

Part (1) of the proposition is a consequence of (3.2) and (3.4).

The proof of part (2) of the proposition follows the same lines.

Let

$\mathcal{F}_{c}\left(a, k_{2}, \ldots, k_{n}\right)=\mathfrak{k}+i\left(\mathcal{T}_{a}+\operatorname{Ad}\left(k_{2}\right) \mathcal{T}_{a}+\operatorname{Ad}\left(k_{2} k_{3}\right) \mathcal{T}_{a}+\cdots+\operatorname{Ad}\left(k_{2} \cdots k_{n}\right) \mathcal{T}_{a}\right)$

and

$$
\mathcal{F}\left(a, k_{2}, \ldots, k_{n}\right)=\mathfrak{k}+\mathcal{T}_{a}+\operatorname{Ad}\left(k_{2}\right) \mathcal{T}_{a}+\operatorname{Ad}\left(k_{2} k_{3}\right) \mathcal{T}_{a}+\cdots+\operatorname{Ad}\left(k_{2} \cdots k_{n}\right) \mathcal{T}_{a} .
$$

COROLlary 3.5 .

(1) $\mathcal{V}\left(a_{c}, k_{2}, \ldots, k_{n}\right) \subseteq \mathcal{F}_{c}\left(a, k_{2}, \ldots, k_{n}\right)$.

(2) $\mathcal{V}\left(a, k_{2}, \ldots, k_{n}\right)=\mathcal{F}\left(a, k_{2}, \ldots, k_{n}\right)$.

REMARK 3.6. The inclusion in (1) of Corollary 3.5 is strict as will be seen in Section 5.

PROOF. (1) By Proposition 3.4, it is enough to prove that

$$
\mathcal{W}\left(a_{c}, k_{2}, \ldots, k_{n}\right) \subseteq \mathcal{F}_{c}\left(a, k_{2}, \ldots, k_{n}\right) .
$$

Then

$$
\operatorname{Ad}\left(k_{2}\right)\left(X_{\alpha}-\theta X_{\alpha}\right)=\sum_{\beta \in \Sigma} c_{\beta}\left(X_{\beta}-\theta X_{\beta}\right)+X
$$


for some $X \in \mathfrak{a}$ depending on $k_{2}$ and $\alpha$. Combining Lemma 3.3 and (3.5) gives

$$
\begin{aligned}
i \operatorname{Ad}\left(a_{c} k_{2}\right)\left(X_{\alpha}-\theta X_{\alpha}\right)= & i \operatorname{Ad}\left(a_{c}\right)\left(\sum_{\beta \in \Sigma} c_{\beta}\left(X_{\beta}-\theta X_{\beta}\right)+X\right) \\
= & i \sum_{\beta \in \Sigma} c_{\beta} \operatorname{Ad}\left(a_{c}\right)\left(X_{\beta}-\theta X_{\beta}\right)+i \operatorname{Ad}\left(a_{c}\right) X \\
= & -\sum_{\beta \in \Sigma} c_{\beta} \sin \beta(H)\left(X_{\beta}+\theta X_{\beta}\right) \\
& \quad+i \sum_{\beta \in \Sigma} c_{\beta} \cos \beta(H)\left(X_{\beta}-\theta X_{\beta}\right)+i X
\end{aligned}
$$

Combining (3.5) and (3.6), we get

$$
\begin{aligned}
& i \operatorname{Ad}\left(a_{c} k_{2}\right)\left(X_{\alpha}-\theta X_{\alpha}\right)-i \operatorname{Ad}\left(k_{2}\right)\left(X_{\alpha}-\theta X_{\alpha}\right) \\
& =-\sum_{\beta \in \Sigma} c_{\beta} \sin \beta(H)\left(X_{\beta}+\theta X_{\beta}\right) \\
& \quad+i \sum_{\beta \in \Sigma} c_{\beta}(\cos \beta(H)-1)\left(X_{\beta}-\theta X_{\beta}\right) \\
& \in \mathfrak{k}+i \mathcal{T}_{a} .
\end{aligned}
$$

Therefore

$$
i \operatorname{Ad}\left(a_{c} k_{2}\right) \mathcal{T}_{a} \subseteq \mathfrak{k}+i\left(\mathcal{T}_{a}+\operatorname{Ad}\left(k_{2}\right) \mathcal{T}_{a}\right) .
$$

Since $\mathcal{T}_{a_{c}} \subseteq \mathcal{T}_{a}$, it follows that

$$
i \operatorname{Ad}\left(a_{c} k_{2}\right) \mathcal{T}_{a_{c}} \subseteq \mathfrak{k}+i\left(\mathcal{T}_{a}+\operatorname{Ad}\left(k_{2}\right) \mathcal{T}_{a}\right)
$$

From (3.7) and (3.8) we deduce that

$$
\begin{aligned}
i \operatorname{Ad}\left(a_{c} k_{2} a_{c} k_{3}\right) \mathcal{T}_{a_{c}} & =\operatorname{Ad}\left(a_{c} k_{2}\right)\left(i \operatorname{Ad}\left(a_{c} k_{3}\right) \mathcal{T}_{a_{c}}\right) \\
& \subseteq \mathfrak{k}+i\left(\mathcal{T}_{a}+\operatorname{Ad}\left(k_{2}\right) \mathcal{T}_{a}+\operatorname{Ad}\left(k_{2} k_{3}\right) \mathcal{T}_{a}\right) .
\end{aligned}
$$

By induction, we get

$$
\mathcal{W}\left(a_{c}, k_{2}, \ldots, k_{n}\right) \subseteq \mathcal{F}_{c}\left(a, k_{2}, \ldots, k_{n}\right) .
$$

Hence part (1) of the corollary.

(2) Using (3.5) and Lemma 3.3,

$$
\begin{aligned}
\operatorname{Ad}\left(a k_{2}\right)\left(X_{\alpha}-\theta X_{\alpha}\right)-\operatorname{Ad}\left(k_{2}\right)\left(X_{\alpha}-\theta X_{\alpha}\right) \\
=\sum_{\beta \in \Sigma} c_{\beta}(\cosh \beta(H)-1)\left(X_{\beta}-\theta X_{\beta}\right) \\
\quad+\sum_{\beta \in \Sigma} c_{\beta} \sinh \beta(H)\left(X_{\beta}+\theta X_{\beta}\right) \in \mathfrak{k}+\mathcal{T}_{a} .
\end{aligned}
$$

Therefore

$$
\operatorname{Ad}\left(a k_{2}\right) \mathcal{T}_{a} \subseteq \mathfrak{k}+\mathcal{T}_{a}+\operatorname{Ad}\left(k_{2}\right) \mathcal{T}_{a} \quad \text { and } \quad \operatorname{Ad}\left(k_{2}\right) \mathcal{T}_{a} \subseteq \mathfrak{k}+\mathcal{T}_{a}+\operatorname{Ad}\left(a k_{2}\right) \mathcal{T}_{a} .
$$


Thus

$$
\mathcal{V}\left(a, k_{2}\right)=\mathcal{F}\left(a, k_{2}\right) .
$$

From arguments similar to Corollary 3.5(1), we obtain

$$
\mathcal{V}\left(a, k_{2}, k_{3}\right) \subseteq \mathcal{F}\left(a, k_{2}, k_{3}\right) .
$$

Also, using (3.9), we get

$$
\begin{aligned}
\mathcal{F}\left(a, k_{2}, k_{3}\right) & \subseteq \mathfrak{k}+\mathcal{T}_{a}+\operatorname{Ad}\left(a k_{2}\right) \mathcal{T}_{a}+\operatorname{Ad}\left(a k_{2}\right) \operatorname{Ad}\left(k_{3}\right) \mathcal{T}_{a} \\
& \subseteq \mathfrak{k}+\mathcal{T}_{a}+\operatorname{Ad}\left(a k_{2}\right) \mathcal{T}_{a}+\operatorname{Ad}\left(a k_{2}\right)\left(\mathfrak{k}+\mathcal{T}_{a}+\operatorname{Ad}\left(a k_{3}\right) \mathcal{T}_{a}\right) \\
& =\mathcal{V}\left(a, k_{2}, k_{3}\right) .
\end{aligned}
$$

Hence

$$
\mathcal{V}\left(a, k_{2}, k_{3}\right)=\mathcal{F}\left(a, k_{2}, k_{3}\right) .
$$

Continuing inductively, we get

$$
\mathcal{V}\left(a, k_{2}, \ldots, k_{n}\right)=\mathcal{F}\left(a, k_{2}, \ldots, k_{n}\right) .
$$

This concludes the proof.

Proposition 3.7. Let $G / K$ be a symmetric space and $a_{1}, \ldots, a_{r} \in G$. Suppose that $v_{a_{1}}, \ldots, v_{a_{r}}$ are the orbital measures on $G$ supported on the double cosets $K a_{1} K, \ldots, K a_{r} K$. Then:

(1) $v_{a_{1}} * \cdots * v_{a_{r}}$ is absolutely continuous with respect to the left Haar measure on $G$ if and only if the function $\Psi$ given by

$$
\begin{aligned}
\Psi: K^{r+1} & \longrightarrow G \\
\left(k_{1}, \ldots, k_{r+1}\right) & \longmapsto k_{1} a_{1} k_{2} \cdots k_{r} a_{r} k_{r+1}
\end{aligned}
$$

has full rank at some point $\left(k_{1}, \ldots, k_{r+1}\right) \in K^{r+1}$;

(2) for $X_{1}, \ldots, X_{r+1} \in \mathfrak{k}$ and $\left(k_{1}, \ldots, k_{r+1}\right) \in K^{r+1}$,

$$
\begin{aligned}
& (d \Psi)_{\left(k_{1}, \ldots, k_{r+1}\right)}\left(X_{1}, \ldots, X_{r+1}\right) \\
& \quad=X_{1}+\operatorname{Ad}\left(k_{1} a_{1}\right) X_{2}+\cdots+\operatorname{Ad}\left(k_{1} a_{1} \cdots k_{r} a_{r}\right) X_{r+1} .
\end{aligned}
$$

Proof. (1) Ragozin [12, Theorem 2.5] proved that if the rank of $\Psi$ is equal to the dimension of $G$ at one point $\left(k_{1}, k_{2}, \ldots, k_{r+1}\right)$ in $K^{r+1}$, then $v_{a_{1}} * \cdots * v_{a_{r}}$ is absolutely continuous. Conversely, if rank $\Psi$ is less than the dimension of $G$ at every $\left(k_{1}, k_{2}, \ldots, k_{r+1}\right)$, then Sard's theorem says the measure of the image of $\Psi$ is zero. But the image is the product of the double cosets, $K a_{1} K \cdots K a_{r} K$, which supports the measure $v_{a_{1}} * \cdots * v_{a_{r}}$, and hence this measure is singular.

(2) Let $X_{1}$ be an element of $T_{k_{1}} K \cong \mathfrak{k}$, and $f$ a smooth function in a neighborhood of $k_{1} a_{1} k_{2} a_{2} \cdots k_{r} a_{r} k_{r+1}$. Then

$$
\begin{aligned}
d \Psi_{\left(k_{1}, \ldots, k_{r+1}\right)}\left(X_{1}, 0, \ldots, 0\right) f & =\left.\frac{d}{d t} f\left(\exp \left(t X_{1}\right) k_{1} a_{1} \cdots k_{r} a_{r} k_{r+1}\right)\right|_{t=0} \\
& =X_{1} f
\end{aligned}
$$


Similarly, for $X_{2}$ an element of $T_{k_{2}} K \cong \mathfrak{k}$, and $f$ as above,

$$
\begin{aligned}
& d \Psi_{\left(k_{1}, \ldots, k_{r+1}\right)}\left(0, X_{2}, \ldots, 0\right) f \\
& \quad=\left.\frac{d}{d t} f\left(k_{1} a_{1} \exp \left(t X_{2}\right) k_{2} a_{2} \cdots k_{r} a_{r} k_{r+1}\right)\right|_{t=0} \\
& \quad=\left.\frac{d}{d t} f\left(\exp \left(\operatorname{Ad}\left(k_{1} a_{1}\right) t X_{2}\right) k_{1} a_{1} k_{2} a_{2} \cdots k_{r} a_{r} k_{r+1}\right)\right|_{t=0} \\
& \quad=\operatorname{Ad}\left(k_{1} a_{1}\right) X_{2} .
\end{aligned}
$$

Hence

$$
d \Psi_{\left(k_{1}, \ldots, k_{r+1}\right)}\left(0, X_{2}, \ldots, 0\right)=\operatorname{Ad}\left(k_{1} a_{1}\right) X_{2} .
$$

By induction, we get

$$
d \Psi_{\left(k_{1}, \ldots, k_{r+1}\right)}\left(0, \ldots, 0, X_{i}, 0, \ldots, 0\right)=\operatorname{Ad}\left(k_{1} a_{1} \cdots k_{i-1} a_{i-1}\right) X_{i} .
$$

Therefore

$$
\begin{aligned}
d \Psi_{\left(k_{1}, \ldots, k_{r+1}\right)}\left(X_{1}, X_{2}, \ldots, X_{r+1}\right) & \\
= & X_{1}+\operatorname{Ad}\left(k_{1} a_{1}\right) X_{2}+\cdots+\operatorname{Ad}\left(k_{1} a_{1} \cdots k_{i-1} a_{i-1}\right) X_{i}+\cdots \\
& +\operatorname{Ad}\left(k_{1} a_{1} \cdots k_{r-1} a_{r-1}\right) X_{r}+\operatorname{Ad}\left(k_{1} a_{1} \cdots k_{r} a_{r}\right) X_{r+1} .
\end{aligned}
$$

This completes the proof.

REMARK 3.8. Note that $d \Psi_{\left(k_{1}, \ldots, k_{r+1}\right)}$ is independent of $k_{r+1}$.

Our next proposition is similar to Proposition 3.7.

PRoposition 3.9. Let $H_{1}, \ldots, H_{r} \in \mathfrak{p}$ and suppose that $\mu_{K, H_{1}}, \ldots, \mu_{K, H_{r}}$ are the orbital measures on $\mathfrak{p}$ supported on the orbits $O_{K, H_{i}}$. Then:

(1) $\mu_{K, H_{1}} * \cdots * \mu_{K, H_{r}}$ is absolutely continuous with respect to the Lebesgue measure on $\mathfrak{p}$ if and only if the function

$$
\begin{aligned}
\psi: O_{K, H_{1}} \times \cdots \times O_{K, H_{r}} & \longrightarrow \mathfrak{p} \\
\left(X_{1}, \ldots, X_{r}\right) & \longmapsto X_{1}+\cdots+X_{r}
\end{aligned}
$$

has full rank at some point $\left(X_{1}, \ldots, X_{r}\right) \in O_{K, H_{1}} \times \cdots \times O_{K, H_{r}}$;

(2) letting $Y \in O_{K, H}$, the tangent space $T_{Y}\left(O_{K, H}\right)$ of $O_{K, H}$ at $Y$ is $\{[X, Y] \mid X \in \mathfrak{k}\}$;

(3) letting $Y_{1}, \ldots, Y_{r} \in \mathfrak{k},\left(X_{1}, \ldots, X_{r}\right) \in O_{K, H_{1}} \times \cdots \times O_{K, H_{r}}$, we have

$$
\begin{aligned}
& (d \psi)_{\left(X_{1}, \ldots, X_{r}\right)}: T_{X_{1}}\left(O_{K, H_{1}}\right) \times \cdots \times T_{X_{r}}\left(O_{K, H_{r}}\right) \longrightarrow \mathfrak{p} \\
& \left(\left[X_{1}, Y_{1}\right], \ldots,\left[X_{r}, Y_{r}\right]\right) \longmapsto\left[X_{1}, Y_{1}\right]+\cdots+\left[X_{r}, Y_{r}\right] \text {. }
\end{aligned}
$$

PROOF. (1) This is similar to the proof of Proposition 3.7.

(2) Let $X \in \mathfrak{k}$. Then the curve $\gamma(t)=\operatorname{Ad}(\exp (t X)) Y$ is inside $O_{K, H}$ and passes through $Y$ at $t=0$. Since the derivative of $\gamma$ at $t=0$ is $[X, Y]$, we deduce that

$$
T_{Y}\left(O_{K, H}\right)=\{[X, Y] \mid X \in \mathfrak{k}\} .
$$

(3) This follows from (2). 
Proof of TheOREM 3.1. (1) Suppose that there exists a positive integer $r$ such that $v_{a_{c}}^{r}$ is absolutely continuous with respect to the Haar measure on $G_{c}$. Then, by Proposition 3.7, we deduce that there exist $\left(k_{1}, k_{2}, \ldots, k_{r}\right)$ such that

$$
\mathfrak{k}+\operatorname{Ad}\left(k_{1} a_{c}\right) \mathfrak{k}+\operatorname{Ad}\left(k_{1} a_{c} k_{2} a_{c}\right) \mathfrak{k}+\cdots+\operatorname{Ad}\left(k_{1} a_{c} \cdots k_{r} a_{c}\right) \mathfrak{k}=\mathfrak{g}_{c} .
$$

The fact that $\operatorname{Ad}\left(k_{1}^{-1}\right) \mathfrak{k}=\mathfrak{k}$, along with the definition of $\mathcal{V}\left(a_{c}, k_{2}, \ldots, k_{n}\right)$, shows that (3.10) is equivalent to

$$
\mathcal{V}\left(a_{c}, k_{2}, \ldots, k_{n}\right)=\mathfrak{g}_{c} .
$$

From Corollary 3.5(1) and (3.11) we get

$$
\mathfrak{g}_{c}=\mathcal{V}\left(a_{c}, k_{2}, \ldots, k_{n}\right) \subseteq \mathcal{F}_{c}\left(a, k_{2}, \ldots, k_{n}\right)
$$

From (3.12) and the definition of $\mathcal{V}\left(a_{c}, k_{2}, \ldots, k_{n}\right)$ it follows that

$$
\mathfrak{g}_{c}=\mathfrak{k}+i\left(\mathcal{T}_{a}+\operatorname{Ad}\left(k_{2}\right) \mathcal{T}_{a}+\operatorname{Ad}\left(k_{2} k_{3}\right) \mathcal{T}_{a}+\cdots+\operatorname{Ad}\left(k_{2} \cdots k_{n}\right) \mathcal{T}_{a}\right) .
$$

Hence

$$
\mathfrak{p}=\mathcal{T}_{a}+\operatorname{Ad}\left(k_{2}\right) \mathcal{T}_{a}+\operatorname{Ad}\left(k_{2} k_{3}\right) \mathcal{T}_{a}+\cdots+\operatorname{Ad}\left(k_{2} \cdots k_{n}\right) \mathcal{T}_{a} .
$$

Consequently

$$
\begin{aligned}
\mathcal{F}\left(a, k_{2}, \ldots, k_{n}\right) & =\mathfrak{k}+\mathcal{T}_{a}+\operatorname{Ad}\left(k_{2}\right) \mathcal{T}_{a}+\operatorname{Ad}\left(k_{2} k_{3}\right) \mathcal{T}_{a}+\cdots+\operatorname{Ad}\left(k_{2} \cdots k_{n}\right) \mathcal{T}_{a} \\
& =\mathfrak{g} .
\end{aligned}
$$

Combining Corollary 3.5(2), and Proposition 3.7(2), we deduce that $v_{a}^{r}$ is absolutely continuous with respect to the left Haar measure on $G$. Therefore $n_{a} \leq n_{a_{c}}$.

(2) $\mu_{K, H}^{r}$ is absolutely continuous with respect to the Lebesgue measure on $\mathfrak{p}$ if and only if there exist points $x_{1}, \ldots, x_{r} \in K$, such that for $i=1, \ldots, r, X_{i}=\operatorname{Ad}\left(x_{i}\right) H$ and

$$
T_{X_{1}}\left(O_{K, H}\right)+\cdots+T_{X_{r}}\left(O_{K, H}\right)=\mathfrak{p} .
$$

Since $T_{X_{i}}\left(O_{K, H}\right)=\operatorname{Ad}\left(x_{i}\right) T_{H}\left(O_{K, H}\right)$ for $i=1, \ldots, r$, Equation (3.13) holds if and only if

$$
\operatorname{Ad}\left(x_{1}\right) T_{H}\left(O_{K, H}\right)+\cdots+\operatorname{Ad}\left(x_{r}\right)\left(O_{K, H}\right)=\mathfrak{p} .
$$

Without loss of generality we can assume that $x_{1}=e$ in the identity (3.13). Let $k_{2}=x_{2}, k_{3}=k_{2}^{-1} x_{2}$ and $k_{r}=k_{r-1}^{-1} \cdots k_{2}^{-1} x_{r}$. Using (3.14), Corollary 3.5 and the observation $T_{H}\left(O_{K, H}\right)=\mathcal{T}_{a}$, we deduce that (3.14) holds if and only if

$$
\mathfrak{k}+\mathcal{T}_{a}+\operatorname{Ad}\left(k_{2}\right) \mathcal{T}_{a}+\cdots+\operatorname{Ad}\left(k_{2} \cdots k_{r}\right) \mathcal{T}_{a}=\mathfrak{g} .
$$

But (3.15) holds if and only if $v_{a}^{r}$ is absolutely continuous with respect to the left Haar measure on $G$. 
The following corollary of Theorem 3.1(2) was proved by second author and Hare in [2, Theorem 4.1].

COROLlary 3.10. Let $G_{c}$ be a compact connected simple Lie group and $\mathfrak{g}_{c}$ be its Lie algebra. Let $\mathfrak{t}$ be a Cartan subalgebra of $\mathfrak{g}_{c}, H \in \mathfrak{t}, a=\exp (i H)$ and $r$ a positive integer. Then $v_{a}^{r}$ is absolutely continuous with respect to the left Haar measure on $G_{c}^{\mathbb{C}}$ if and only if $\mu_{H}^{r}$ is absolutely continuous with respect to Lebesgue measure on $\mathfrak{g}_{c}$.

PROOF. Consider the noncompact symmetric space $G_{c}^{\mathbb{C}} / G_{c}$. In this case $\mathfrak{p}$ is $i \mathfrak{g}_{c}$ and $\mathfrak{a}$ is $i \mathrm{t}$. Therefore the corollary follows from Theorem 3.1(2).

\section{Transference of results}

In this section we transfer results of the second author with Hare, mentioned in the introduction, to noncompact symmetric spaces. Before we state our first transference result, we need the following definition.

Definition 4.1. Given a restricted $\operatorname{root} \alpha \in \Sigma$ and $a \in \exp \mathfrak{a}$, say $a=\exp (H)$ for $H \in \mathfrak{a}$, we set $\alpha(a)=\alpha(H)$ (this is well defined, as exp is a diffeomorphism from $\mathfrak{a}$ onto $\exp (\mathfrak{a}))$. We call the element $a \in \exp \mathfrak{a}$ regular if $\alpha(a) \neq 0$ for all $\alpha \in \Sigma$. We say that $a_{c}=\exp (i H)$ is regular if $\alpha(a) \neq 0(\bmod \pi)$ for all $\alpha \in \Sigma$. It is clear from the definitions of regular elements that if $a_{c}$ is regular then $a$ is also regular.

The following theorem is proved in [4, Theorem 3.1].

THEOREM 4.2. Suppose that $A, B \in \mathfrak{a}$, and put $a_{c}=\exp (i A)$ and $b_{c}=\exp (i B)$. If $a_{c}$ and $b_{c}$ are regular elements in $G_{c}$ and $v_{a_{c}}, v_{b_{c}}$ are the associated $K$-orbital measures, then $v_{a_{c}} * v_{b_{c}}$ is absolutely continuous with respect to the Haar measure on $G_{c}$.

Using Theorems 3.1(1) and 4.2, we have the following analogue of Theorem 4.2 on noncompact symmetric spaces.

Theorem 4.3. Let $A, B \in \mathfrak{a}, \quad a=\exp (A), b=\exp (B)$, and let $v_{a}, v_{b}$ be the associated $K$-orbital measures on $G$. If $a$ and $b$ are regular elements in $G$ then $v_{a} * v_{b}$ is absolutely continuous with respect to the left Haar measure on $G$.

PROOF. Let $t_{0} \in \mathbb{R}$ be such that $a_{c}\left(t_{0}\right)=\exp \left(i t_{0} A\right)$ and $b_{c}\left(t_{0}\right)=\exp \left(i t_{0} B\right)$ are regular elements in $G_{c}$. Then by Theorem 4.2, $v_{a_{c}\left(t_{0}\right)} * v_{b_{c}\left(t_{0}\right)}$ is absolutely continuous with respect to the Haar measure on $G_{c}$. Therefore by Theorem 3.1(1), $v_{a\left(t_{0}\right)} * v_{b\left(t_{0}\right)}$ is absolutely continuous on $G$ with respect to the left Haar measure on $G$. Hence by Theorem 3.1(2), $\mu_{K, t_{0} A} * \mu_{K, t_{0} B}$ is absolutely continuous with respect to the Lebesgue measure on $\mathfrak{p}$. So, $O_{K, t_{0} A}+O_{K, t_{0} B}=t_{0}\left(O_{K, A}+O_{K, B}\right)$ contains an open set in it and therefore $\left(O_{K, A}+O_{K, B}\right)$ has an open set in it. Hence $\mu_{K, A} * \mu_{K, B}$ is absolutely continuous with respect to the Lebesgue measure on $\mathfrak{p}$. Therefore $v_{a} * v_{b}$ is absolutely continuous with respect to the left Haar measure on $G$ by Theorem 3.1(2). 
For the next result, take $G=\mathrm{SL}(n, \mathbb{R})$ and $K=\mathrm{SO}(n)$. Then $G_{c}=\mathrm{SU}(n)$. For $a \in G \backslash N_{G}(K)$ (respectively $a_{c} \in G_{c} \backslash N_{G_{c}}(K)$ ), Ragozin [12, Theorem 2.5] had shown that $v_{a}^{r} \in L^{1}(G)$ (respectively $v_{a_{c}}^{r} \in L^{1}\left(G_{c}\right)$ ) for $r=\operatorname{dim} G / K=\left(n^{2}+n-2\right) / 2$. He had speculated that $\operatorname{dim} G / K$ is not sharp, but had conjectured that (approximately) $r=n / 2$ would work [12, p. 375]. Improving upon the result of Ragozin, the following result was proved in [6, Theorem 3.1].

THEOREM 4.4. If $A \in \mathfrak{a}, a=\exp (A)$, and $a_{c}$ is not in the normalizer of $\mathrm{SO}(n)$ in $\mathrm{SU}(n)$, then $v_{a_{c}}^{n}$ is absolutely continuous with respect to the Haar measure on $\mathrm{SU}(n)$.

Using Theorems 3.1(1) and 4.4, we obtain the following analogue of Ragozin's result for the noncompact symmetric space $\operatorname{SL}(n, \mathbb{R}) / \mathrm{SO}(n)$.

THEOREM 4.5. If $A \in \mathfrak{a}, a=\exp (A)$, and $a$ is not in the normalizer of $\mathrm{SO}(n)$ in $\operatorname{SL}(n, \mathbb{R})$, then $v_{a}^{n}$ is absolutely continuous with respect to the left Haar measure on $\mathrm{SL}(n, \mathbb{R})$.

PROOF. The proof is similar to the proof of Theorem 4.3 and is left to the reader.

\section{Final remarks}

The aim of this section is to show that the inequality in Theorem 3.1(1) can be strict. For this let $G_{c}=\operatorname{SO}(2 n+1)$, the group of $(2 n+1) \times(2 n+1)$ orthogonal matrices of determinant 1 . Then the complexification $G_{c}^{\mathbb{C}}$ of $G_{c}$ is equal to

$$
\mathrm{SO}(n, \mathbb{C})=\left\{g \in \mathrm{SL}(n, \mathbb{C}): g^{t} g=1\right\} .
$$

As was explained in Section 2.1, the dual of the compact Lie group $G_{c}=\mathrm{SO}(2 n+1)$, seen as a symmetric space, is $G_{c}^{\mathbb{C}} / G_{c}$. Let $E_{i j}$ denote the $(2 n+1) \times(2 n+1)$ matrix with $(i, j)$ th entry 1 and all other entries zero. Set $H=\sum_{i=1}^{n} i \pi\left(E_{2 i-1,2 i}-E_{2 i, 2 i-1}\right)$, $a=\exp (H)$ and $a_{c}=\exp (i H)$.

It is shown in [5, Theorem 6.8] (respectively [7, Theorem 8.2] or [3, Theorem 6.6]) that $v_{a_{c}}^{(2 n-1)}$ is singular with respect to the Haar measure on $G_{c}$, (respectively $\mu_{i H}^{n}$ is absolutely continuous with respect to Lebesgue measure on the Lie algebra $\mathfrak{s o}(2 n+1)$ of $\mathrm{SO}(2 n+1))$.

Also, it follows from Corollary 3.10 that $v_{a}^{n}$ is absolutely continuous with respect to the left Haar measure on $\mathrm{SO}(n, \mathbb{C})$ if and only if $\mu_{i H}^{n}$ is absolutely continuous with respect to Lebesgue measure on $\mathfrak{s o}(2 n+1)$. Therefore $v_{a}^{n}$ is absolutely continuous with respect to the left Haar measure on $\mathrm{SO}(n, \mathbb{C})$ but $v_{a_{c}}^{n}$ is singular with respect to the Haar measure on $\mathrm{SO}(2 n+1)$. This shows that inequality in Theorem 3.1(1) can be strict.

Open Problem. Let $G / K$ be a noncompact symmetric space. Let $H \in \mathfrak{a}$ and $r$ be a positive integer. It is shown in [2, Theorem 3.1] that for a noncompact symmetric space $G_{c}^{\mathbb{C}} / G_{c}, \mu_{\mathfrak{g}_{c}, H}^{r} \in L^{2}\left(\mathfrak{g}_{c}\right)$ if and only if $\nu_{\exp (H)}^{r} \in L^{2}\left(G_{c}^{\mathbb{C}}\right)$. It is an open problem whether $\mu_{K, H}^{r} \in L^{2}(\mathfrak{p})$ if and only if $v_{\exp (H)}^{r} \in L^{2}(G)$. 


\section{Acknowledgement}

The second author would like to thank Kathryn Hare for showing the proof of Theorem 4.3 .

\section{References}

[1] C. Dunkl, 'Operators and harmonic analysis on the sphere', Trans. Amer. Math. Soc. 125 (1966), 250-263.

[2] S. Gupta and K. Hare, 'Singular dichotomy for orbital measures on complex groups', Boll. Unione Mat. Ital. (9) III (2010), 409-419.

[3] S. Gupta and K. Hare, 'Singularity of orbits in classical Lie algebras', Geom. Funct. Anal. 13 (2003), 815-844.

[4] S. Gupta and K. Hare, 'Convolutions of generic orbital measures in compact symmetric spaces', Bull. Aust. Math. Soc. 79(3) (2009), 513-522.

[5] S. Gupta and K. Hare, ' $L^{2}$-singular dichotomy for orbital measures of classical compact Lie groups', Adv. Math. 222 (2009), 1521-1573.

[6] S. Gupta and K. Hare, 'Smoothness of convolution powers of orbital measures on the symmetric space SU(n)/SO(n)', Monatsh. Math. 159 (2010), 27-43.

[7] S. Gupta, K. Hare and S. Seyfaddini, ' $L^{2}$-singular dichotomy for orbital measures of classical simple Lie algebras', Math. Z. 262 (2009), 91-124.

[8] S. Helgason, Differential Geometry, Lie Groups and Symmetric Spaces (Academic Press, New York, 1978).

[9] A. W. Knapp, Lie Groups: Beyond an Introduction (Birkhäuser, Boston, 2002).

[10] M. Mimura and H. Toda, Topology of Lie Groups, Translations of Mathematical Monographs, 91 (American Mathematical Society, Providence, RI, 1991).

[11] D. Ragozin, 'Central measures on compact simple Lie groups', J. Funct. Anal. 10 (1972), 212-229.

[12] D. Ragozin, 'Zonal measure algebras on isotropy irreducible homogeneous spaces', J. Funct. Anal. 17 (1974), 355-376.

[13] V. S. Varadarajan, Lie Groups and Lie Algebras and their Representations (Springer, New York, 1984).

BOUDJEMÂA ANCHOUCHE, Department of Mathematics and Statistics, Sultan Qaboos University, PO Box 36, Al-Khoud 123, Muscat, Sultanate of Oman e-mail: anchouch@squ.edu.om

SANJIV KUMAR GUPTA, Department of Mathematics and Statistics, Sultan Qaboos University, PO Box 36, Al-Khoud 123, Muscat, Sultanate of Oman e-mail: gupta@squ.edu.om 